\title{
INVERSÃO GRAVIMÉTRICA 3D DE CORPOS SALINOS NA BACIA DE CAMPOS UTILIZANDO DADOS DE SENSORIAMENTO REMOTO
}

\author{
3D GRAVIMETRIC INVERSION OF BODIES OF SALT IN THE CAMPOS BASIN USING \\ REMOTE SENSING DATA
}

\author{
Fabio Ullmann Furtado de LIMA ${ }^{1}$, Leila Maria Véspoli de CARVALHO ${ }^{2}$ \\ ${ }^{1}$ Centro de Ensino Superior da Foz do Itajaí - Univ. do Estado de Santa Catarina (UDESC), Balneário Camboriú - SC , Brasil. \\ fuflima@gmail.com \\ ${ }^{2}$ Dept. of Geography of University of California Santa Barbara (UCSB), EUA. leila.vespoli@gmail.com

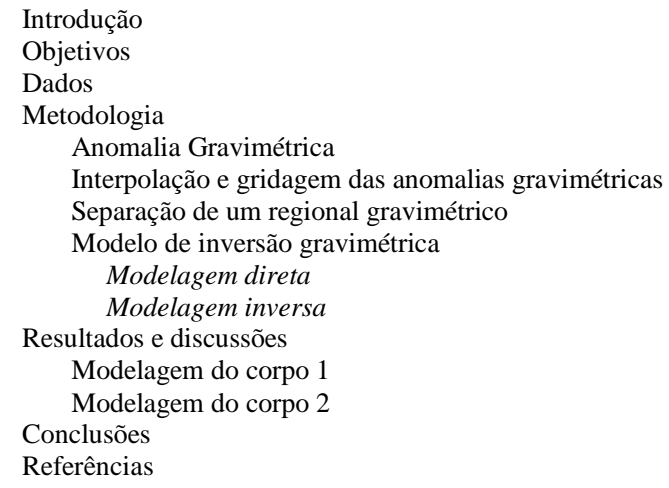

RESUMO - Foi realizada a inversão tridimensional de dados gravimétricos oriundos de sensoriamento remoto com o objetivo de estimar as geometrias em sub-superfície de dois corpos salinos inseridos na Bacia de Campos. As modelagens dos corpos são baseadas em informações a priori tais como: contrastes de densidade entre corpos e o meio, números de prismas e números de vértices de cada prisma que, superpostos, modelarão a forma dos corpos salinos, profundidades e distâncias radiais mínimas e máximas dos corpos, coordenadas de origem dos prismas (a partir das quais o modelo começará a estimar os corpos salinos), entre outros. Todas estas informações a priori provêm de um ensaio sísmico de reflexão realizados na região de estudo. Foram modelados paredões de sal considerando dois casos distintos: uma anomalia residual Bouguer aberta (caso 1) e outra fechada (caso 2). As geometrias dos paredões de sal foram modeladas satisfatoriamente em ambos os casos. Entretanto, o tamanho do paredão no caso 1 teve que ser subestimado para se obter um melhor ajuste entre as isogais observadas e preditas. Os resultados mostram que o modelo de inversão gravimétrica 3D parece mais eficiente em modelar anomalias Bouguer fechadas do que abertas.

Palavras-chave: gravimetria, inversão 3D, corpos salinos, Bacia de Campos.

ABSTRACT - Tridimensional inversion of gravimetric data from remote sensing was conducted to estimate subsurface geometries of 2 bodies of salt in the Campos Basin. The modeling of the bodies are based on a priori informations like the density contrast between the bodies and the environment, number of prisms and number of vertex of each prism that, superimposed, are able to give the form of the bodies of salt, depths, minimum and maximum radial distances of the bodies, origin coordenates of the prisms (from which the model will begin to construct the bodies of salt), and so on. All these a priori informations come from reflexion seismics profiles executed in the region of studies. Bodies of salt were modeled considering two distincts cases: an opened residual Bouguer anomaly (case 1) and another closed (case 2). The geometries of the bodies of salt were well modeled in both cases, as compared to the seismics profiles. However, the size of the first body of salt was underestimated in order to obtain a better ajustment between the predicted and observed isogals. Thus, the 3D gravimetric inversion model seems to be more eficiente in modeling closed Bouguer anomalies than opened ones.

Keywords: gravimetry, 3D inversion, salt bodies, Campos Basin.

\section{INTRODUÇÃO}

A Bacia de Campos é uma bacia de margem passiva formada durante o Neocomiano (Cretácio Inferior $\sim 135 \mathrm{Ma}$ ), associada ao evento de ruptura do supercontinente Gondwana. Sua área total é de aproximadamente $115800 \mathrm{~km}^{2}$, sendo limitada ao sul pelo Alto de Cabo Frio (Bacia de Santos) e ao norte pelo Alto de Vitória (Bacia do Espírito Santo). Em 2007, possuía 47 campos petrolíferos, com produção de 1,46 x $10^{6}$ barris/dia de petróleo e 21,97 $\mathrm{m}^{3} /$ dia de gás (ANP, 2007), sendo uma das bacias petrolíferas brasileiras mais prolíficas (OJEDA, 1982; MOHRIAK et al., 1987, 1990; CONTRERAS et al., 2010).

Os métodos gravimétricos e magnetométricos têm sido amplamente utilizados como métodos iniciais para prospecção geofísica, pois os equipamentos podem ser aerotransportados, permitindo ampla cobertura de dados em regiões de difícil acesso, como no caso da Bacia de Campos. Além disso, a altimetria por satélite 
também vem sendo amplamente utilizada e tem extrema importância em estudos de regiões de difícil acesso. Apesar de os métodos gravimétricos apresentarem baixa resolução quando comparada à resolução do método sísmico, estes métodos são eficazes em detectar corpos e feições geológicas que apresentem contraste lateral de densidade em relação ao meio em que estão inseridos. Na prospecção por hidrocarbonetos, a gravimetria tem sido utilizada para localizar falhas verticais no embasamento de bacias sedimentares e na detecção de corpos salinos. Entretanto, a correta interpretação de dados de gravimetria pode consistir em um problema (em casos de má aplicações dos dados ou interpretações equivocadas), visto que ela não apresenta uma solução única. Neste caso, um problema desse tipo pode ser contornado através da incorporação de informações geológicas $a$ priori sobre as fontes anômalas em profundidade, compensando a insuficiência de informação dos dados de forma satisfatória.

A utilização de anomalias gravimétricas para a reconstrução da geometria de corpos geológicos em subsuperfície é um problema que vem sendo investigado por modelagem direta iterativa e inversão (TALWANI et al., 1959; CORDELL \& HENDERSON, 1968; BARBOSA \& SILVA, 1994; BARBOSA et al., 1997; DUTRA \& MARANGONI, 2009; OLIVEIRA \& BARBOSA, 2013). A principal dificuldade na utilização da modelagem direta interativa é a complicada tarefa de testar uma grande variedade de geometrias (2D ou 3D) por tentativa e erro até encontrar uma que represente as anomalias de gravidade de maneira aceitável (STARICH et al., 1994; NAGIHARA \& HALL, 2001).

A utilização da inversão para a reconstrução de massas anômalas em subsuperfície pode ser dividida em dois grupos. O primeiro considera a distribuição dos contrastes de densidade das massas anômalas variável e estima essa distribuição dos contrastes de densidade; e o segundo estima a forma geométrica dos corpos, considerando fixo o constraste de densidade das massas anômalas. O primeiro grupo, em geral, parametriza a região em subsuperfície por meio de uma malha de prismas retangulares 2D ou 3D (retângulos ou cubos) e justapostos nas direções dos eixos Cartesianos ( $\mathrm{x}$ e $\mathrm{y}$ ou $\mathrm{x}, \mathrm{y}$ e $\mathrm{z}$ ). O contraste de densidade dentro de cada prisma é a variável a ser determinada e as informações $a$ priori são as dimensões de cada prisma. Deste modo, os contrastes de densidade em cada prisma são os parâmetros desconhecidos do problema inverso e os corpos geológicos em subsuperfície são reconstruídos por meio da estimativa dos mesmos (BEAR et al., 1995; LI \& OLDENBURG, 1998; NAGIHARA \& HALL, 2001; KRAHENBUHL \& LI, 2006).

Neste trabalho, será utilizado um método de inversão sob a premissa do conhecimento do contraste de densidade entre a massa anômala e o meio. Assim, o objetivo aqui é unicamente de estimar a forma do corpo, com a informação $a$ priori da constituição deste corpo (densidades do corpo e do meio conhecidas). A metodologia que será utilizada neste trabalho foi desenvolvida por OLIVEIRA JUNIOR (2010) sendo este um método de reconstrução de corpos 3D baseado em uma generalização do método de inversão radial 2D desenvolvido por SILVA \& BARBOSA (2004). O método aproxima o corpo geológico $3 \mathrm{D}$ por múltiplos prismas retos, justapostos verticalmente e com seções horizontais descritas por polígonos, cujos vértices são descritos em coordenadas polares referidas a uma origem que se localiza arbitrariamente dentro desse polígono. A reconstrução do corpo geológico é feita pela estimativa dos raios dos vértices e das coordenadas da origem do polígono que descreve a seção horizontal de cada prisma. Nessa estimativa, empregam-se vínculos matemáticos que introduzem informações geológicas sobre a forma do corpo.

Os métodos de inversão gravimétrica têm sido utilizados por diversos autores para a reconstrução da geometria de corpos salinos ou na determinação da distribuição de densidade em subsuperfície (STARICH et al., 1994; NAGIHARA \& HALL, 2001). O sal representa um grande obstáculo na prospecção por hidrocarbonetos utilizando sísmica de reflexão, pois há grande atenuação da energia da onda refletida, problemas de ambiguidade na interpretação sísmica e problemas devido a múltiplas reflexões (OEZSEN, 2004). Para contornar estes problemas, foram publicados nos últimos anos alguns casos de sucesso na aplicação de métodos potenciais como um colaborador aos métodos sísmicos (SILVA DIAS et al., 2011). 


\section{OBJETIVOS}

Deseja-se utilizar dados de gravimetria por satélite com o objetivo de identificar a geometria em sub superfície de corpos salinos inseridos ao longo de um perfil na Bacia de Campos. Informações a priori que serão utilizadas como parâmetros no modelo de inversão serão determinadas a partir de um perfil sísmico de reflexão que fornecerá informações sobre as localizações dos corpos salinos e das profundidades dos topos e base dos corpos. Além disso, estes perfis sísmicos focalizados sobre os corpos de sal a serem modelados servirão para verificar a eficiência do modelo de inversão em simular tridimensionalmente tais corpos salinos, já que nos próprios perfis é possível identificar as geometrias dos corpos salinos bidimensio- nalmente. Este trabalho visa mostrar que estudos de gravimetria em bacias sedimentares com potencial petrolífero podem ser úteis para estabelecer os volumes relativos das rochas de sal utilizando contrastes de densidade conhecidos. Além disso, uma determinação satisfatória da geometria dos corpos salinos é de grande valia na prospecção de hidrocarbonetos. Isto porque a análise permite inferir a localização de reservatórios, visto que a dinâmica do sal no tempo geológico pode influenciar nos processos de migração e armazenamento dos hidrocarbonetos em regiões preferenciais pela combinação de armadilhas estruturais e estratigráficas criadas pela movimentação do sal.

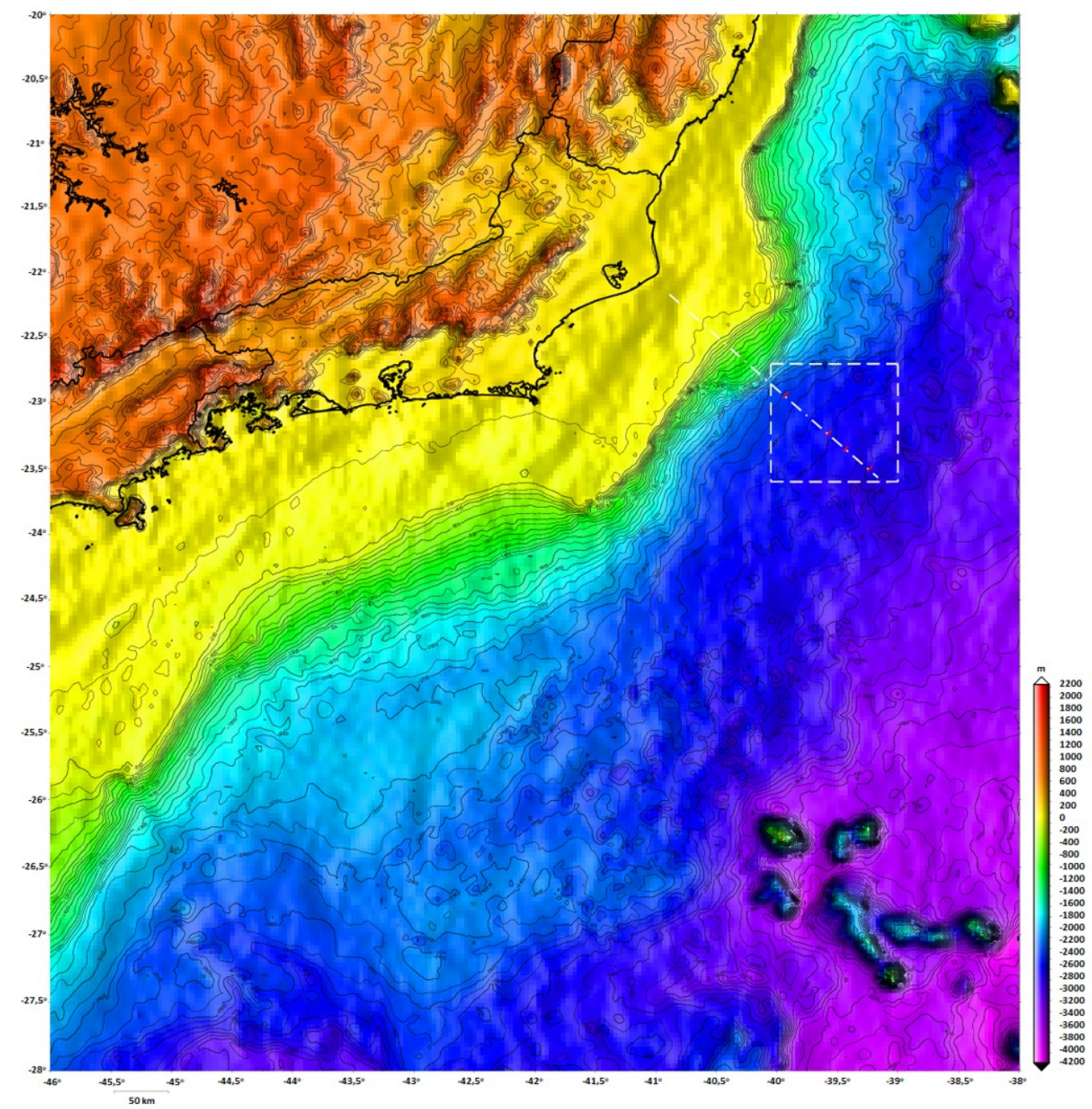

Figura 1: Mapa da topografia interpolada utilizando splines para a região da Bacia de Campos e parte da Bacia de Santos (isolinhas de altitude em metros). Dados oriundos de altimetria por satélites e batimetria marinha. O intervalo de contorno é de $200 \mathrm{~m}$.

\section{DADOS}

Dados de gravimetria e topografia foram são compilações oriundas de altimetria por utilizados neste trabalho. Os dados em questão satélites, gravimetria e batimetria marinha, 
sendo disponibilizados pela University of California San Diego (UCSD) em: http://topex.ucsd.edu/ cgi-bin/get_data.cgi (SMITH \& SANDWELL, 1997; SANDWELL \& SMITH, 2009; SANDWELL et al., 2013, 2014). Os dados foram obtidos para uma região que compreende a Bacia de Campos e a parte norte da Bacia Santos, cujos extremos coordenados são: $\left(46^{\circ} \mathrm{W}, 20^{\circ} \mathrm{S}\right)$ e $\left(38^{\circ} \mathrm{W}, 28^{\circ} \mathrm{S}\right)$. Na figura 1 , o retângulo que compreende as longitudes entre $40.05^{\circ} \mathrm{W}$ e $39^{\circ} \mathrm{W}$ e as latitudes entre $22.70{ }^{\circ} \mathrm{S}$ e $23.60{ }^{\circ} \mathrm{S}$ mostra a região de estudo que contém os dois corpos salinos a serem modelados. O perfil traçado dentro do retângulo na figura 1 (em frente ao Cabo de São Tomé - RJ) coincide com a seção sísmica da linha R de MOHRIAK et al. (2008) (figura 2). A linha R da figura 2 tem cerca de $264 \mathrm{Km}$, enquanto que o perfil traçado na figura 1 tem cerca de $235 \mathrm{Km}$. Tais discrepâncias nos comprimentos das linhas pode ser explicado pela utilização de interpolação dos dados originais de satélite utilizando splines, necessário para se obter os dados em ponto de grade. A profundidade da região de estudo compreende aproximadamente entre $1800 \mathrm{~m}$ e $3100 \mathrm{~m}$ (figura 1). No mapa de isópacas de sal (figura 2) observase que as maiores espessuras do sal coincidem com o final da linha R (região de estudo).

A figura 3, obtida de MOHRIAK et al. (2008), mostra a seção sísmica de reflexão migrada em tempo da região de estudo (perfil inserido dentro do retângulo da figura 1 e linha $\mathrm{R}$ da figura 2). Observamos na figura 3 três corpos salinos, com 2 muralhas de sal limitando sub-bacias na parte direita da seção. Ambas as muralhas de sal supracitadas serão os alvos deste trabalho.

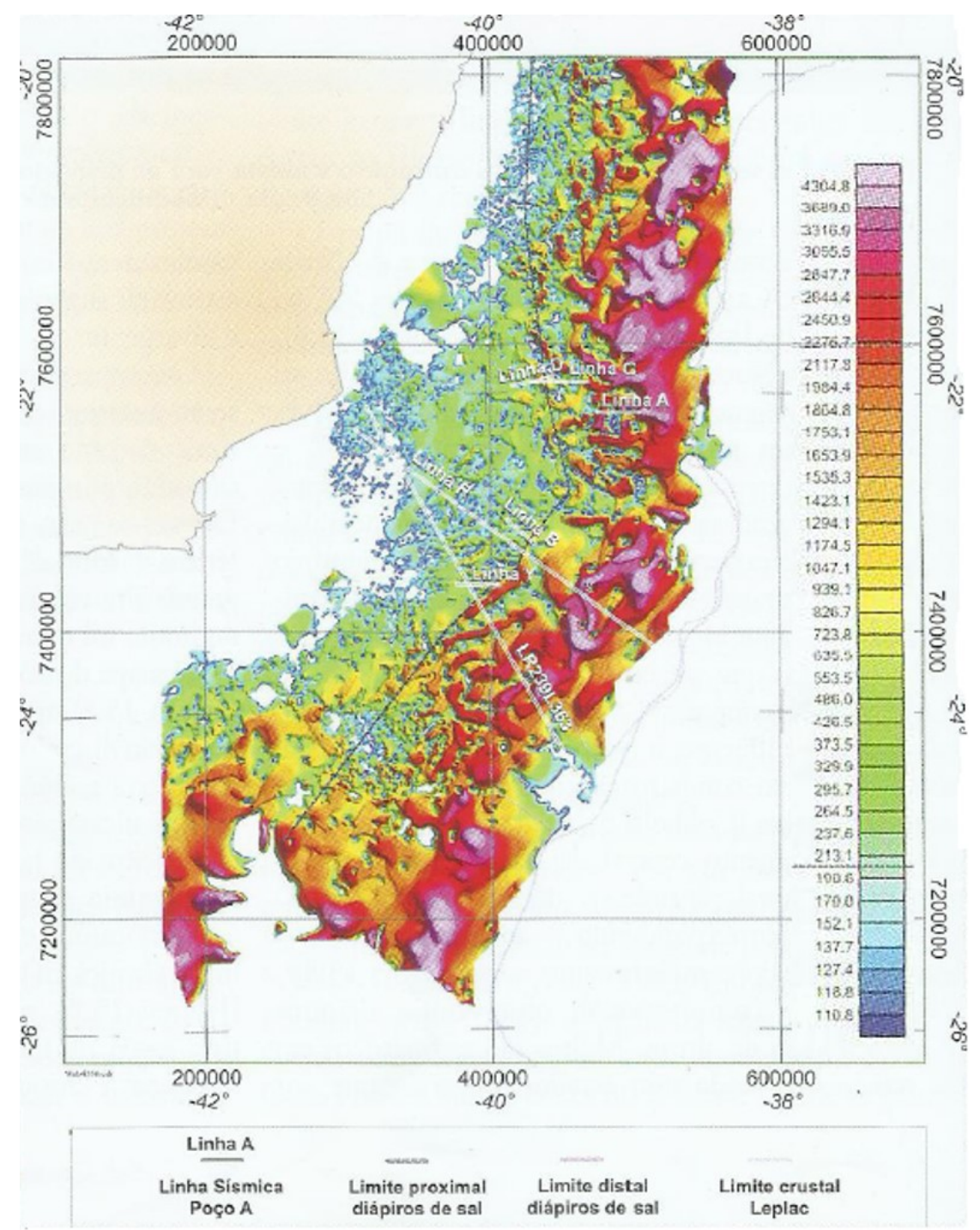

Figura 2: Mapa de isópacas de sal em tempo duplo obtido em MOHRIAK et al. (2008). Linhas tracejadas em preto e cinza claro correspondem aos limites das províncias de sal, segundo DEMERCIAN et al. (1993). A linha pontilhada corresponde ao limite entre crosta continental e oceânica. Os segmentos identificados em linha branca correspondem às seções sísmicas e geológicas discutidas em MOHRIAK et al. (2008), incluindo a linha R discutida no texto. 


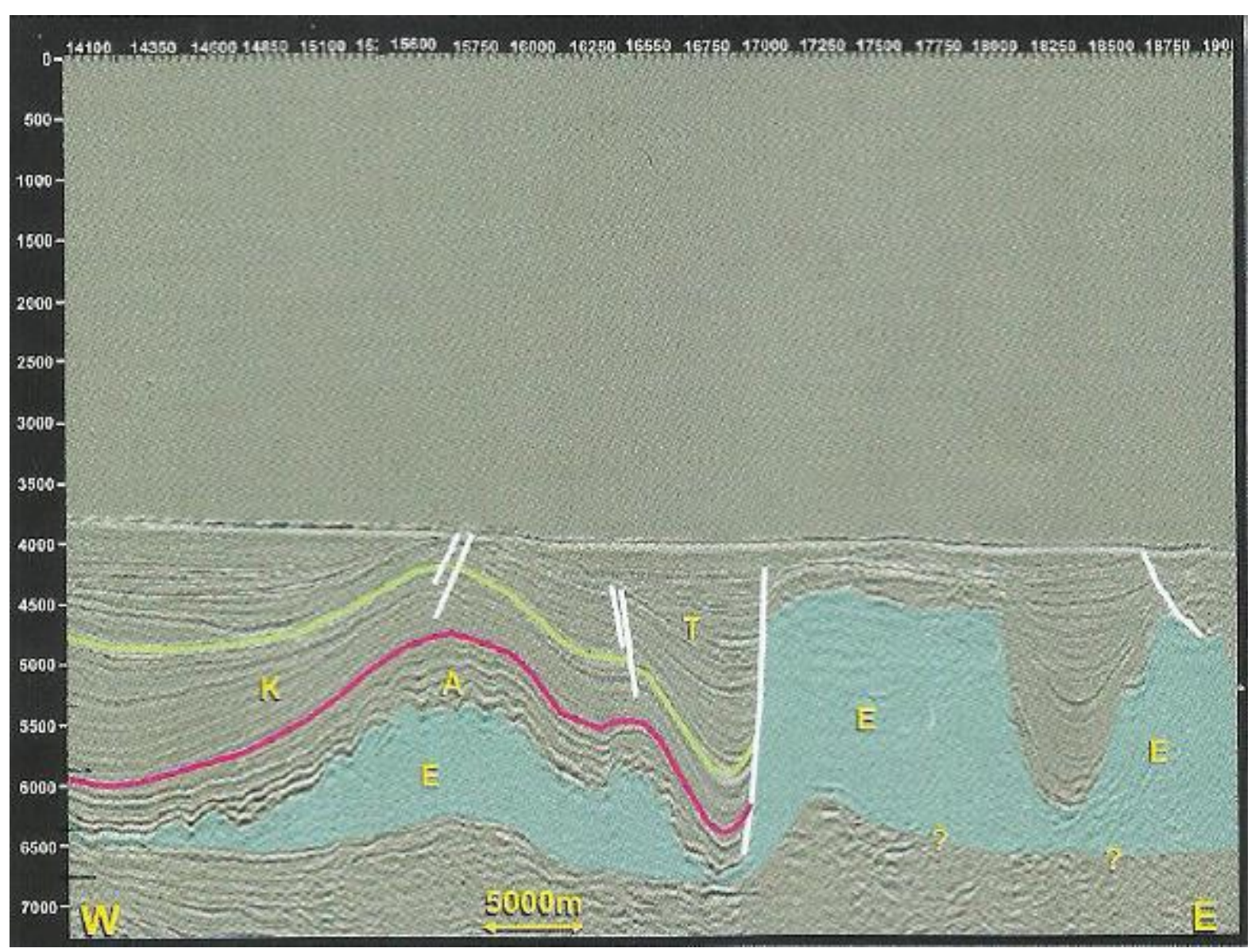

Figura 3: Seção sísmica migrada em tempo. Profundidades da água variam de $2892 \mathrm{~m}$ (PT 13850) a $3120 \mathrm{~m}$ (PT 19000). As unidades estratigráficas são: Terciário (T), Cretáceo (K), Albiano (A) e Evaporitos (E). Domínio compressional com dobras de crescimento de pequeno comprimento de onda afetando os sedimentos do Albiano (entre o topo dos evaporitos e o refletor rosa escuro) e de grande comprimento de onda afetando as sequências do Cretácio Superior e do Terciário. Na parte direita da seção, observar muralhas de sal limitando sub-bacias (minibacias). Obtido de MOHRIAK et al. (2008).

\section{Anomalias gravimétricas}

O processamento inicial dos dados gravimétricos bem como as confecções de mapas será realizado utilizando o programa Generic Mapping Tools (WESSEL \& SMITH, 2008). Os dados de gravidade $g$ disponibilizados pela UCSD já consistem em anomalias ar-livre. A anomalia gravimétrica ar-livre desconsidera os efeitos da altitude nas medidas de $g$ efetuadas em diferentes locais. Ou seja, a correção ar-livre considera a hipótese de que o ponto onde a medida de $g$ foi efetuada seja transportado para um nível de referência pré-estabelecido. Em geral, o nível de referência adotado é o nível médio do mar (NMM) de um oceano não perturbado. Entretanto, somente a aplicação da correção ar-livre nos dados de $g$ implica que a estrutura de massa (topografia regional) seja mantida. Para que o efeito da massa da topografia seja removido dos dados, realiza-se a correção Bouguer.
A anomalia Bouguer $\Delta g_{b}$ é uma correção feita nos dados gravimétricos que considera a massa entre o geóide e a superfície física do local onde foi realizada a medição de $g$. O cálculo de $\Delta g_{b}$ considera uma placa ou plateau de Bouguer semi-infinito com espessura $h$ e densidade $\rho$ (TELFORD et al., 1990; BLAKELY, 1996). Geralmente, utiliza-se o valor médio de $\rho$ para a crosta continental terrestre $\left(\rho_{\text {crosta }}=2.67 \mathrm{~g} \cdot \mathrm{cm}^{-3}\right)$. Considerando a força de atração exercida pelo plateau de Bouguer sobre um ponto de massa unitária sobre sua superfície, verifica-se que a mesma é dada por $2 \pi G \rho h$, sendo $G$ a constante gravitacional universal. Considerando $G$ como $6.67 \times 10^{-11} \mathrm{~m}^{3} \cdot \mathrm{kg}^{-1} \cdot \mathrm{s}^{-2}$, chega-se a um fator de correção para $\Delta g_{b}$ igual à $0.1119 H$, onde $H$ é a altutitude ortométrica (distância vertical entre o geóide e a superfície física do ponto de medição) medida em metros. Assim, calcula-se $\Delta g_{b}$ como em (1), sendo $\gamma$ um valor 
padrão de gravidade dado como em (2) e o termo $0.3086 H$ a correção ar-livre. Em (2), $\varphi$ é a latitude (em graus) do ponto da medida.
Os resultados obtidos pelas equações (1) e (2) são dados em $\mathrm{mGal}$, sendo $1 \mathrm{Gal}$ igual a 1 $\mathrm{cm} \cdot \mathrm{s}^{-2}$.

$$
\begin{gathered}
\Delta g_{b}=g-\gamma-0.3086 H-0.1119 H(1) \\
\gamma=978031.846\left(1+0.005278895 \operatorname{sen}^{2} \varphi-0.000023 \operatorname{sen}^{4} \varphi\right.
\end{gathered}
$$

Em dados marinhos, a correção Bouguer calcula a carência de massa existente entre o assoalho oceânico e o NMM, como se as regiões marinhas fossem preenchidas de rochas da crosta ao invés da água do mar. Neste caso, a correção Bouguer é dada como em (3), onde $\rho_{\text {crosta }}$ é o valor padrão de densidade da rocha com a qual o mar deveria estar preenchido, $\rho_{\text {água }}=1.003 \mathrm{~g} . \mathrm{cm}^{-3}$ é o valor padrão da água do mar (que está preenchendo a camada) e $h$ corresponde à batimetria no ponto considerado.

$$
\text { Corr. Bouguer }=2 \pi G\left(\rho_{\text {crosta }}-\rho_{\text {água }}\right) h
$$

\section{Interpolação e gridagem das anomalias gravimétricas}

O segundo processamento dos dados utilizando o GMT consistiu na interpolação e gridagem das anomalias gravimétricas. Anteriormente ao processo de interpolação e gridagem, as anomalias geralmente são uniformizadas espacialmente pela remoção através da média de alguns dados que se apresentavam muito próximos uns dos outros. Em geral, o algoritmo mais comum utilizado para a interpolação e a gridagem de dados utiliza splines de curvatura contínua sob tensão (comando surface no GMT). Um spline é uma curva definida matematicamente por dois ou mais pontos de controle. Os pontos de controle que ficam na curva são chamados de nós (RUGGIERO \& LOPES, 1997). Neste trabalho, para fins comparativos, também foram utilizados algoritmos de interpolação e gridagem que efetuam a média entre pontos vizinhos, fornecendo um valor médio para cada nó que tem um ou mais pontos inseridos dentro de um raio de uma circunferência centrada sobre ele (comando nearneighbor no GMT); e algoritmos que encontram o melhor meio de conectar os pontos de modo a encontrar a melhor triangulação equilátera possível (comando triangulate no GMT).

\section{Separação de um regional gravimétrico}

$\mathrm{O}$ passo posterior à interpolação e gridagem das anomalias Bouguer consiste na separação de um regional para a obtenção da anomalia Bouguer residual. Geralmente, o regional é associado a feições mais profundas, suaves e de maior comprimento de onda. Já o residual destaca as anomalias gravimétricas associadas a feições mais superficiais, ou seja, de menor comprimento de onda. Assim, as anomalias residuais são obtidas a partir da remoção de um regional, ou seja, da subtração entre a anomalia Bouguer e o regional.

Existem diversas formas de se obter um regional das anomalias Bouguer, dentre as quais se destacam ajustes polinomiais (curvas que melhor se ajustam às isogais de anomalias Bouguer) e a continuação para cima. Neste trabalho, serão ajustados às isogais retas, curvas quadráticas e cúbicas (polinômios de grau 1, 2 e 3, respectivamente).

Basicamente, a continuação para cima usando a Transformada de Fourier vê o campo de uma variável como se as fontes de anomalias estivessem a uma profundidade maior. Assim, a continuação para cima funciona como um filtro que atenua anomalias de curtos comprimentos de onda, servindo perfeitamente para a obtenção de um regional (a interpolação de pontos de grade pode gerar anomalias irreais de curtos comprimentos de onda). A diferença entre as técnicas de filtragem utilizando a remoção de polinômios e a remoção de um regional dado pela continuação para cima é que ao se trabalhar com a Transformada de Fourier, permitem-se deixar embutido no regional as propriedades físicas do campo potencial, diferentemente dos regionais dados por ajuste polinomial. Aqui, os regionais obtidos pela continuação para cima foram calculados utilizando a Transformada Rápida de Fourier ou Fast Fourier Transform $(F F T)$. A FFT é uma operação matemática que transforma uma variável qualquer do domínio do espaço ou do tempo para o domínio do número de onda ou da frequência, respectivamente. A Transformada inversa de Fourier retorna a variável para o domínio do espaço ou do tempo. A Transformada de Fourier 
funciona para campos que representem uma função periódica; entretanto, o campo gravimétrico não é representado por uma função periódica. Neste caso, utiliza-se a FFT por questões de simplificação, pois supondo que o campo gravimétrico seja periódico, cálculos que antes seriam complicados se transformam em simples multiplicações. Em outras palavras, o cálculo da FFT nada mais é do que calcular seus coeficientes aproveitando a propriedade de ortogonalidade de senos e cossenos. O método de simples cálculo das transformadas de Fourier discretas é computacionalmente ineficiente, de maneira que a vantagem da FFT nesses cálculos é em termos logarítmicos. Além disso, com o auxílio da FFT é possível separar as profundidades das fontes. A FFT é usualmente descrita em termos de notação exponencial complexa de Euler (eq. 4), onde $i$ é um número imaginário $(i=\sqrt{-1})$ e $w=2 \pi k / n$, é a frequência angular, sendo $k$ o número de onda e $n$ o número de pontos da série de dados e $z$ a direção no eixo vertical. As anomalias Bouguer residuais são obtidas da subtração entre a anomalia Bouguer e a continuação para cima.

$$
e^{i w z}=\cos (w z)+i \operatorname{sen}(w z)
$$

\section{Modelo de inversão gravimétrica Modelagem direta}

A partir de um conjunto de dados e de conhecimentos pré-existentes a respeito do comportamento de dada propriedade física, é possível obter um modelo da distribuição desta propriedade em sub superfície. Neste trabalho, será determinada a geometria de corpos salinos inseridos numa bacia sedimentar, ou seja, suas distribuições de massa (densidade) em sub superfície cujo comportamento é regido pela teoria do potencial (BLAKELY, 1996). O modelo hipotético visa aproximar os dados preditos (pelo modelo teórico) aos dados observados (medidos), sendo os dados preditos como sendo função de alguns parâmetros (5) (OLIVEIRA JR. \& UIEDA, 2012). Por tentativa e erro, são geradas diferentes geometrias associadas às possíveis distribuições da propriedade física de interesse em sub superfície. A geometria ideal a ser escolhida como a que melhor representa a distribuição da propriedade física de uma feição geológica será aquela em que os dados preditos melhor se ajustam aos dados observados.

$$
\text { dados preditos }=f(\text { parâmetros })
$$

Modelagem inversa

O modelo inverso visa aperfeiçoar, ou seja, aproximar ao máximo os dados preditos aos dados observados utilizando a norma euclidiana para quantificar a distância entre predição e observação (NAGIHARA \& HALL, 2001). Este problema recebe o nome de problema inverso. A figura 4 ilustra a diferença entre o modelo direto e o modelo inverso.

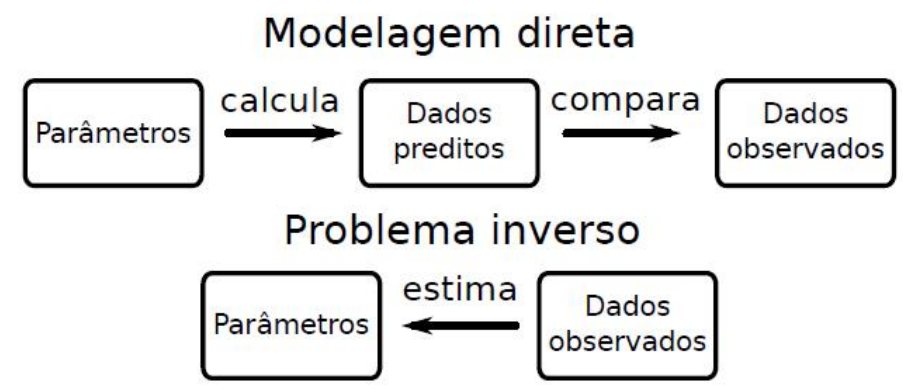

Figura 4: Esquema ilustrativo de modelagem direta e modelagem inversa. Obtido de OLIVEIRA JR. \& UIEDA (2012).

O problema inverso pode ser linear ou não linear (e.g. TALWANI et al. (1959). No caso do problema inverso linear, que será tratado neste trabalho, a função $f(p)$ que correlaciona os parâmetros aos dados preditos pode ser escrita como uma combinação linear dos parâmetros (eq. 6). Na equação $6, f_{i}(p)$ é a função do vetor de parâmetros $p$ calculada para o i-ésimo dado predito $g_{i j}$, com $j=1,2, \ldots, M$ e $b_{i}$ são constantes; $p_{j}$ é o j-ésimo parâmetro.

$$
f_{i}(p)=g_{i 1} p_{1}+g_{i 2} p_{2}+\cdots+g_{i M} p_{M}+b_{i}
$$

A maioria dos problemas geofísicos reais apresentam problemas como instabilidades ou não unicidade de solução. A instabilidade é gerada quando uma pequena alteração dos dados observados causa grandes variações nos parâmetros. A não unicidade ocorre quando um 
mesmo conjunto de parâmetros produz o mesmo conjunto de dados preditos. Neste caso, temos um problema mal posto. Para solucionar o problema mal posto, são estabelecidas restrições através dos chamados vínculos. Os vínculos são baseados em informações a priori da geologia da região em estudo. As informações a priori são introduzidas na resolução dos problemas através de um parâmetro de regularização, que quantifica qual o peso que será dado ao ajuste e qual o peso que será dado à informação $a$ priori (BARBOSA \& SILVA, 1994; BARBOSA et al., 1997; LI \& OLDENBURG, 1998; SILVA \& BARBOSA, 2006; OLIVEIRA \& BARBOSA, 2013).

Neste trabalho, será utilizado um método de inversão gravimétrica para estimar a geometria de corpos geológicos tridimensionais sob a premissa do conhecimento da profundidade do topo e do contraste de densidade entre o corpo e o meio. O método é uma generalização do método de inversão radial $2 \mathrm{D}$ desenvolvido por SILVA \& BARBOSA (2004). Uma descrição completa do modelo pode ser visto em OLIVEIRA JR. (2010). A seguir, será descrito de forma breve o método de inversão que permitirá a modelagem tridimensional de corpos salinos da Bacia de Campos.

Segundo OLIVEIRA JR. (2010), a região que contém o corpo em subsuperfície é discretizada em um conjunto de prismas $3 \mathrm{D}$, retos $\mathrm{e}$ justapostos na direção vertical de um sistema de coordenadas Cartesianas; a espessura e o contraste de densidade dos prismas são conhecidos, contudo, suas seções horizontais são descritas por polígonos com formato desconhecido; as coordenadas horizontais dos vértices desses polígonos representam aproximadamente o contorno das seções horizontais da fonte geológica 3D em diferentes profundidades; cada polígono possui um número fixo de vértices igualmente espaçados de $0^{\circ}$ a $360^{\circ}$, que são descritos em coordenadas polares referidas a uma origem localizada dentro do polígono. O método estima as distâncias radiais associadas aos vértices $\mathrm{e}$ as coordenadas Cartesianas horizontais da origem em cada polígono; a estimativa desses parâmetros por meio de dados gravimétricos permite recuperar um conjunto de prismas verticalmente justapostos com seções horizontais que representam um conjunto de fatias (ou lâminas) horizontais da fonte geológica 3D em diferentes profundidades (OLIVEIRA JR. 2010).

Para obter estimativas estáveis, OLIVEIRA JR. (2010) impôs vínculos matemáticos na estimativa da forma da fonte, que permitem estimar fontes verticais ou inclinadas, isométricas ou não. A estimativa, embora seja estável e ajuste os dados, depende da profundidade máxima adotada para o conjunto de prismas do modelo interpretativo. Para reduzir a classe de possíveis soluções compatíveis com a anomalia de gravidade e com os vínculos, o autor desenvolveu um critério baseado em uma medida dos desajustes dos dados gravimétricos e na massa de estimativas obtidas em sucessivas inversões, em que se adotam diferentes valores para a profundidade máxima do conjunto de prismas 3D justapostos. Este critério consiste em plotar uma curva da massa das estimativas (w) contra as respectivas medidas de desajustes dos dados (s) para vários valores diferentes da profundidade máxima. O valor de profundidade máxima que produz o menor valor da medida de desajuste $s$ na curva $\mathrm{w}$ versus $\mathrm{s}$ é a melhor estimativa da profundidade máxima verdadeira (ou mínima) para a base da fonte, dependendo se os dados têm ou não resolução para recuperar a fonte geológica. Este critério foi deduzido teoricamente pelo autor a partir do teorema de Gauss.

\section{RESULTADOS E DISCUSSÕES}

O primeiro processamento realizado nos dados de anomalias ar-livre disponibilizados pela UCSD foi verificar o melhor tipo de interpolação e gridagem dos mesmos. Os dados foram interpolados utilizando splines, interpolação por vizinhos próximos e triangulação de Delaunay. Verificou-se que o método numérico de interpolação e gridagem por splines apresentou a melhor distribuição espacial dos dados, sem perda de resolução ou problemas de perdas nas bordas, sendo o método adotado (não mostrado).

As anomalias Bouguer foram obtidas segundo a metodologia descrita no item 4.1. Na figura 5, as anomalias foram interpoladas em uma malha regular usando-se splines; e o retângulo delimitado pelas longitudes de $-39,00^{\circ}$ e $-40,05^{\circ}$ e pelas latitudes de $-22,70^{\circ}$ e $-23,60^{\circ}$ representa a região onde estão inseridos os 3 corpos salinos da linha R (MOHRIAK et al., 2008). 


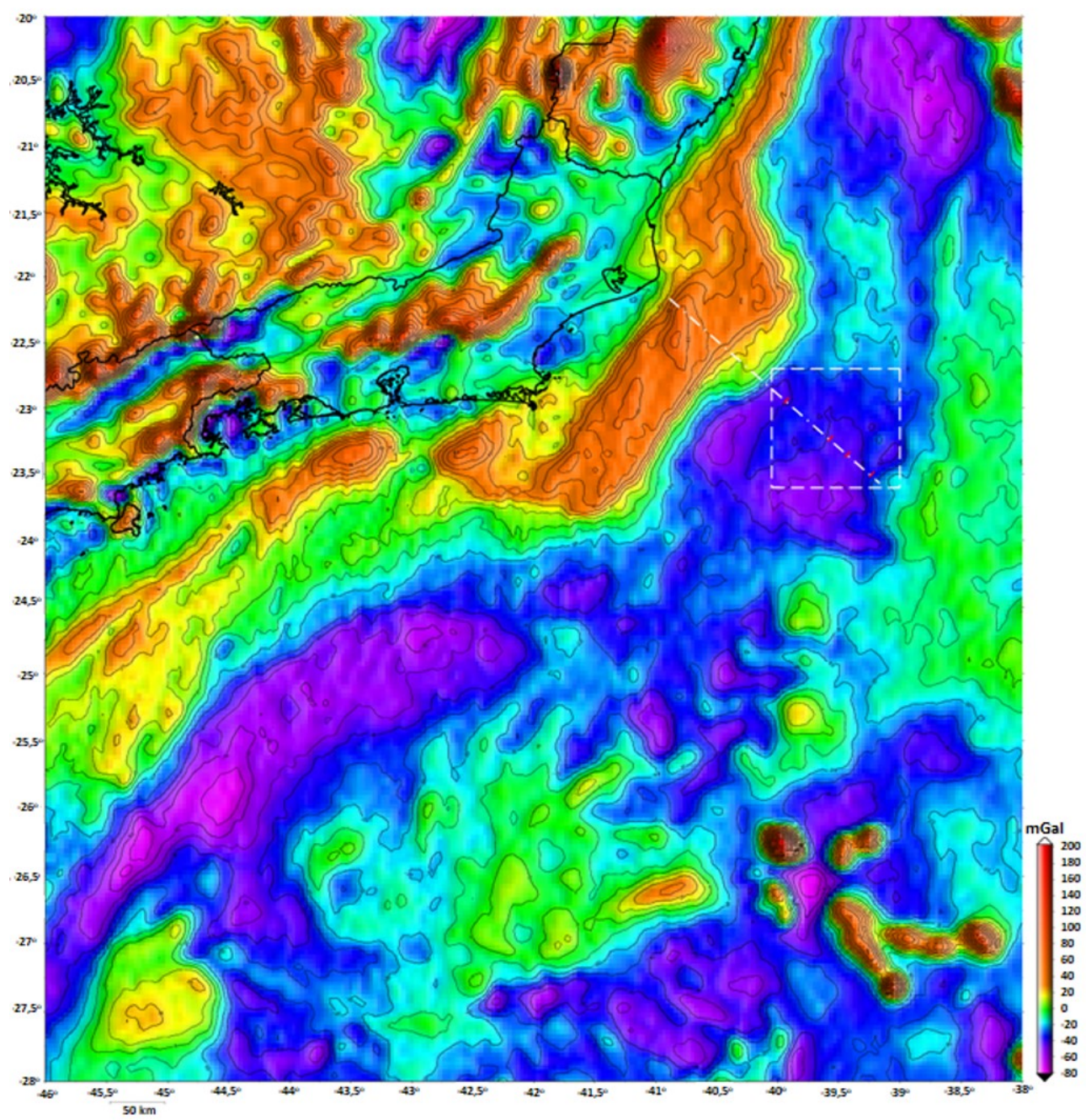

Figura 5: Mapa da anomalia Bouguer interpolada utilizando splines para a região da Bacia de Campos e parte da Bacia de Santos. A linha branca tracejada coincide com a linha R de MOHRIAK et al. (2008) (ver fig. 2) e os traços vermelhos marcados sobre esta marcam posições de mínimos gravimétricos. O intervalo de contorno é de $10 \mathrm{mGal}$.

As bacias sedimentares de Campos e Santos são identificadas por anomalias negativas em zonas submersas, logo após as anomalias positivas associadas à plataforma continental (crosta continental afinada). Além disso, as anomalias mais negativas coincidem com as regiões que apresentam maiores espessuras de sal (ver figura 2). Dentro do retângulo, as anomalias Bouguer mais baixas são valores da ordem de 50 mGal (parte sudoeste do retângulo). O corpo de sal 1 (figura 3) apresenta 2 mínimos gravimétricos da ordem de $-40 \mathrm{mGal}$, enquanto que os corpos de sal 2 e 3 (focos deste trabalho - figura 3) apresentam mínimos gravimétricos da ordem de $-44 \mathrm{mGal}$ (marcas vermelhas no perfil tracejado dentro do retângulo - figura 5).

Os painéis superiores da figura 6 mostram os regionais obtidos pelos ajustes polinomiais de graus 1, 2 e 3 (linear, quadrático e cúbico) à malha da anomalia Bouguer e seus respectivos residuais, obtidos da subtração entre a anomalia
Bouguer e os regionais. Já a figura 7 mostra o regional obtido pela continuação $20 \mathrm{~km}$ para cima (à esquerda) e seu residual (á direta). Os três residuais da figura 6 apresentam anomalias em torno de 0 e $-40 \mathrm{mGal}$; enquanto que as anomalias residuais obtidas da retirada da continuação $20 \mathrm{~km}$ para cima mostram-se mais positivas, em torno de 20 e -20 mGal (figura 7). $\mathrm{Na}$ figura 8 vemos os perfis de anomalias Bouguer original e residuais tomados ao longo da linha branca tracejada da figura 5 (linha R da figura 2). Podemos confirmar a partir desta figura que o residual linear se apresenta sendo o mais negativo dentre todos os residuais. Dito isto, o regional eleito como o melhor foi àquele que gerou residuais mais negativos e com menor comprimento de onda (negativos mais definidos), ou seja, o regional dado por ajuste linear às anomalias Bouguer.

Em primeira instância, efetuou-se a conversão dos dados do sistema cartesiano para 

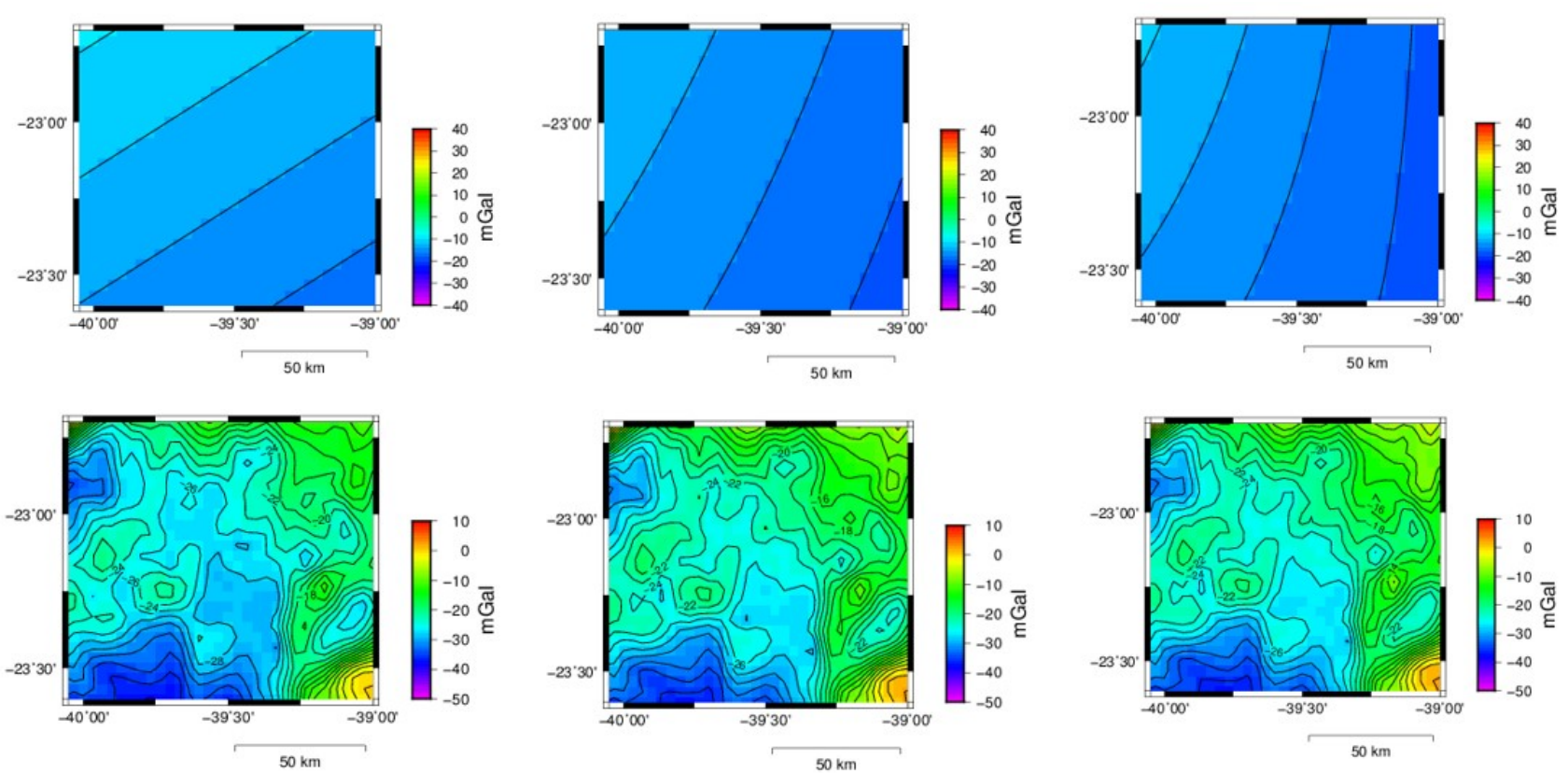

Figura 6: Mapas de anomalias Bouguer regionais (painéis superiores) e seus respectivos residuais (painéis inferiores). Da esquerda para a direita: ajustes polinomiais de grau 1, 2 e 3 . O intervalo de contorno é de 2 mGal, tanto para os regionais como para os residuais.
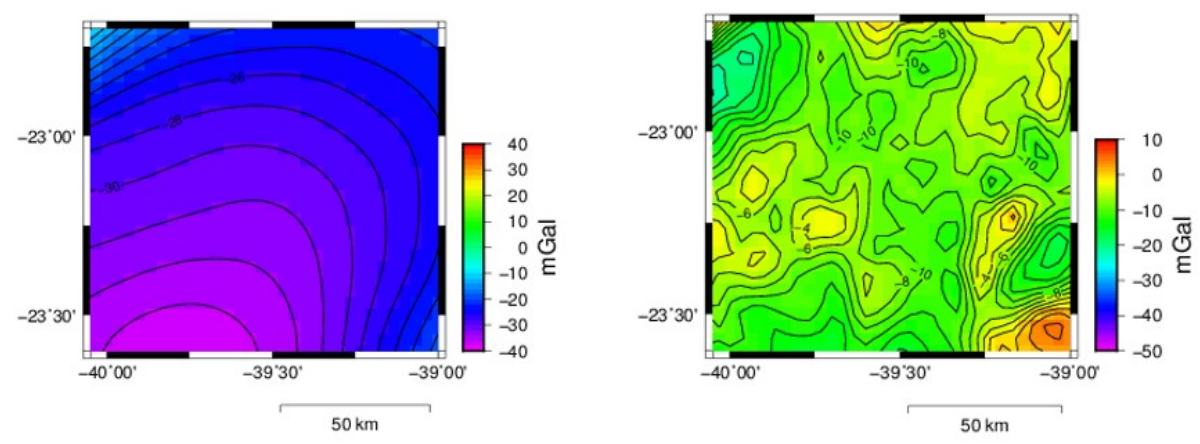

Figura 7: Mapa de anomalia Bouguer regional (painel esquerdo) e seu respectivo residual (painel direito) usando a continuação $20 \mathrm{~km}$ para cima. O intervalo de contorno é de $2 \mathrm{mGal}$, tanto para o regional como para o residual

Perfis Residuais

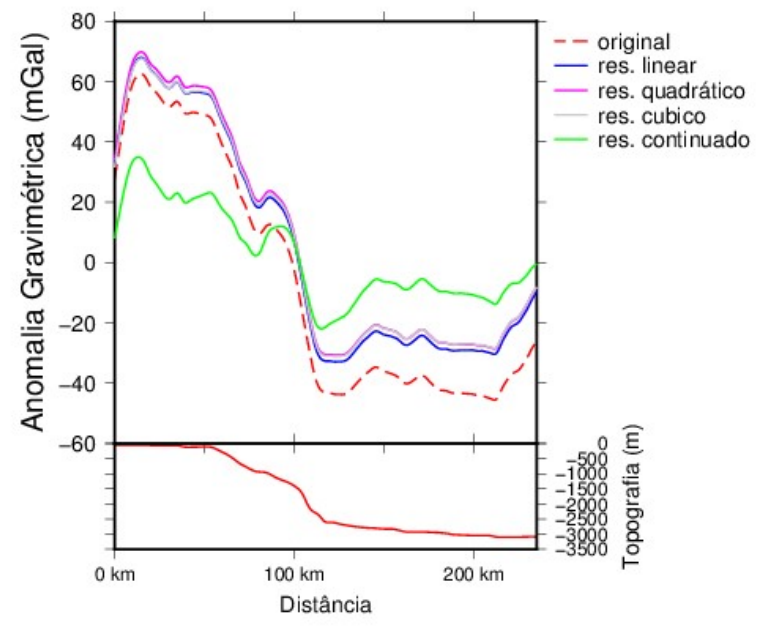

Figura 8: Perfis de anomalias Bouguer inseridos dentro do retângulo limitado pelas longitudes de $-39,00^{\circ}$ e $-40,05^{\circ}$; e pelas latitudes de $-22,70^{\circ}$ e $-23,60^{\circ}$ (linha pontilhada da figura 5).

o sistema UTM (datum de Córrego Alegre, zona modelo uma malha regular interpolada de 24, HS), pois a entrada das coordenadas no anomalias Bouguer residuais limitada pelas modelo de inversão deve ser fornecida em $\mathrm{km}$. longitudes entre $-39,30^{\circ}$ e $-39,80^{\circ}$ e $-23,00^{\circ}$ e Utilizou-se como parâmetro de entrada do $23,60^{\circ}$ para modelagem do paredão 1; e $-39,00^{\circ}$ 
e $-39,30^{\circ}$ e $-23,20^{\circ}$ e $-23,60^{\circ}$ para modelagem do paredão 2 (figura 9). Poderíamos ainda incluir como parâmetro de entrada do modelo as coordenadas UTM dos contornos geológicos de alguma parte aflorante do corpo, se estas estivessem disponíveis (que não é o caso deste trabalho).

O constraste de densidade $\Delta \rho$ entre corpo e meio foi calculado considerando o corpo de sal como composto de halita $\left(\rho_{\text {halita }}=\right.$ $2.22 \mathrm{~g} . \mathrm{cm}^{-3}$ ) e o meio como a rocha geradora, ou seja, folhelho $\left(\rho_{\text {folhelho }}=2.68 \mathrm{~g} \cdot \mathrm{cm}^{-3}\right)$.

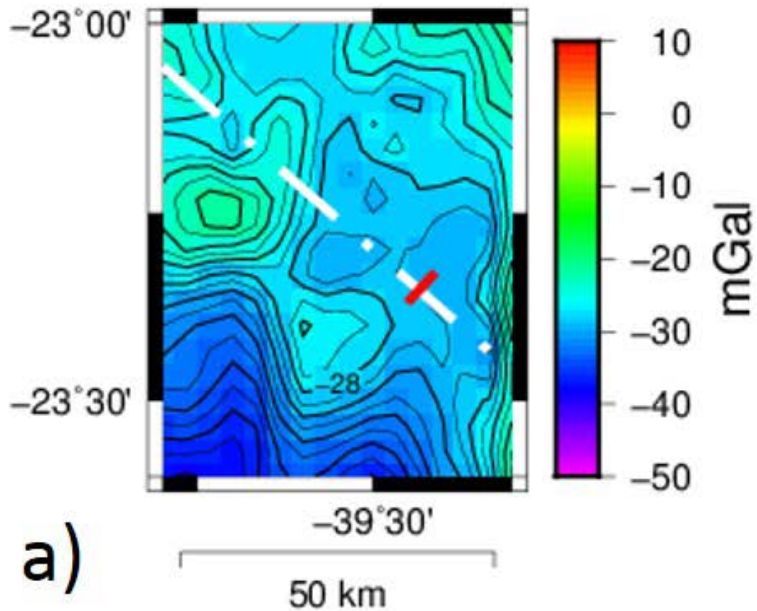

Assim, $\quad\left(\Delta \rho=\rho_{\text {halita }}-\rho_{\text {folhelho }}=2.22-\right.$ $\left.2.68=-0.46 \mathrm{~g} \cdot \mathrm{cm}^{-3}\right)$. O valor de $\Delta \rho$ é coerente com a realidade, visto que $\rho_{\text {halita }}$ varia com as impurezas entre $[2.11 ; 2.22] \mathrm{g}_{\mathrm{cm}} \mathrm{cm}^{-3}$, sendo seu valor típico de 2.22 g. $\mathrm{cm}^{-3}$ (ODÉ 1968, MOHRIAK et al., 2008); e o meio pode consistir de rochas como folhelho, siltito $\left(\rho_{\text {siltito }}=2.67 \mathrm{~g} . \mathrm{cm}^{-3}\right)$, arenito $\left(\rho_{\text {arenito }}=\right.$ $\left.2.66 \mathrm{~g} . \mathrm{cm}^{-3}\right), \quad$ lamito $\quad\left(\rho_{\text {arenito }}=\right.$ $\left.2.69 \mathrm{~g} . \mathrm{cm}^{-3}\right)$ ou carbonatos $\left(\rho_{\text {carbonatos }}=\right.$ $2.70 \mathrm{~g} . \mathrm{cm}^{-3}$ ) (DE MELO GARCIA et al., 2012).

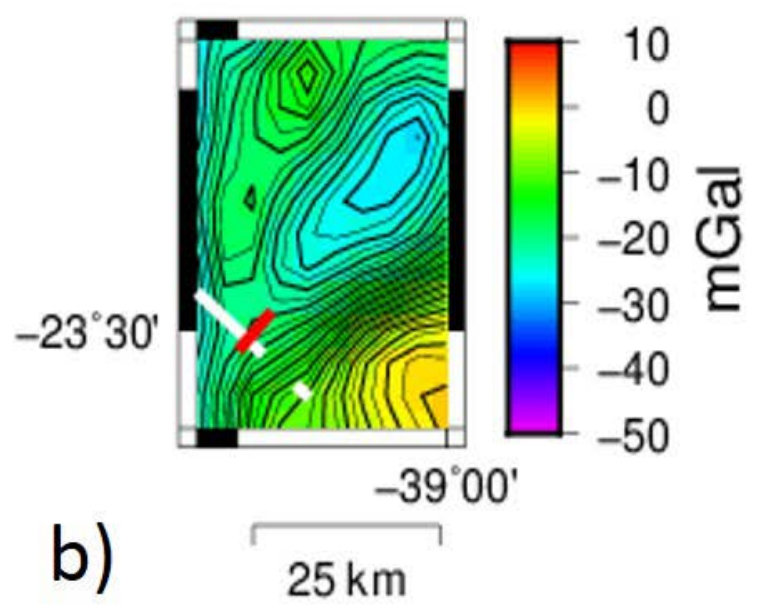

Figura 9: Mapas de anomalias Bouguer residuais obtidos pela retirada de um regional dado por um ajuste linear (polinômio de grau 1) às anomalias Bouguer. Os dados limitados pelas regiões em (a) e (b) foram utilizados como parâmetro de entrada para modelar os paredões de sal 1 (anomalia aberta) e 2 (anomalia fechada), respectivamente. A linha branca tracejada coincide com a linha R de MOHRIAK et al. (2008) (ver figura 2) e os traços vermelhos marcados sobre esta marcam posições de mínimos gravimétricos mostrados ao final dos perfis da figura 8 (após as anomalias Bouguer positivas que representam a quebra de plataforma). O intervalo de contorno é de $1 \mathrm{mGal}$.

\section{Modelagem do corpo 1}

A descrição feita aqui será para os resultados do paredão de sal que apresenta anomalias residuais Bouguer abertas (caso 1), classificado como paredão 1. A figura 10 mostra as coordenadas do mínimo absoluto de anomalia residual Bouguer (quadrado vermelho) a partir das quais os paredões de sal começarão a ser construídos, bem como as coordenadas dos vínculos de desigualdade (triângulos verdes). As coordenadas UTM do mínimo absoluto são (448.985, 7389.934), enquanto que as coordenadas dos vínculos de desigualdade são (445.584, 7389.922), sendo esta referente ao segundo mínimo absoluto; e a outra (452.315, 7412.098).

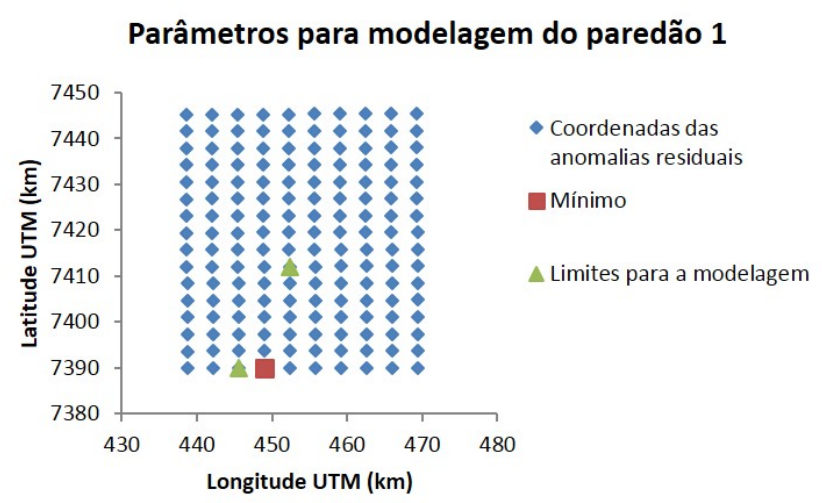

Figura 10: Ponto de mínimo $\left(x_{0}, y_{0}\right)$ a partir do qual os prismas (paredão de sal 1) começarão a ser construídos (quadrado vermelho) e coordenadas limites para a modelagem ou vínculos de desigualdade (triângulos verdes). 
Os vínculos de desigualdade impõem que os parâmetros satisfaçam a desigualdade (7), ou seja, que cada parâmetro estimado $m_{i}$ esteja contido em um intervalo definido pelo intérprete (OLIVEIRA JR., 2010). Esse intervalo pode ser estabelecido com base no conhecimento sobre a geologia da região em estudo ou simplesmente por meio da extensão horizontal da anomalia de gravidade (caso deste trabalho). Em (7), $P$ é o número de parâmetros estimados, que é igual ao número de observações $N$. Se $m_{i}$ for uma distância radial $r_{j}^{k}$, $m_{i \min }=r_{\min }$ e $m_{i \max }=r_{\max }$, para qualquer $j$ e $k$; se $m_{i}$ for a coordenada $x_{0}^{k}$ da origem do $k$ ésimo prisma, $m_{i \min }=x_{0 \min }$ e $m_{i \max }=$ $x_{0 \text { max }}$, para qualquer $k$; finalmente, se $m_{i}$ for a coordenada $y_{0}^{k}$ da origem do $k$-ésimo prisma, $m_{i \min }=y_{0 \min }$ e $m_{i \max }=y_{0 \max }$, para qualquer $k$.

$$
m_{i \min }<m_{i}<m_{i \max }, i=1, \ldots, P
$$
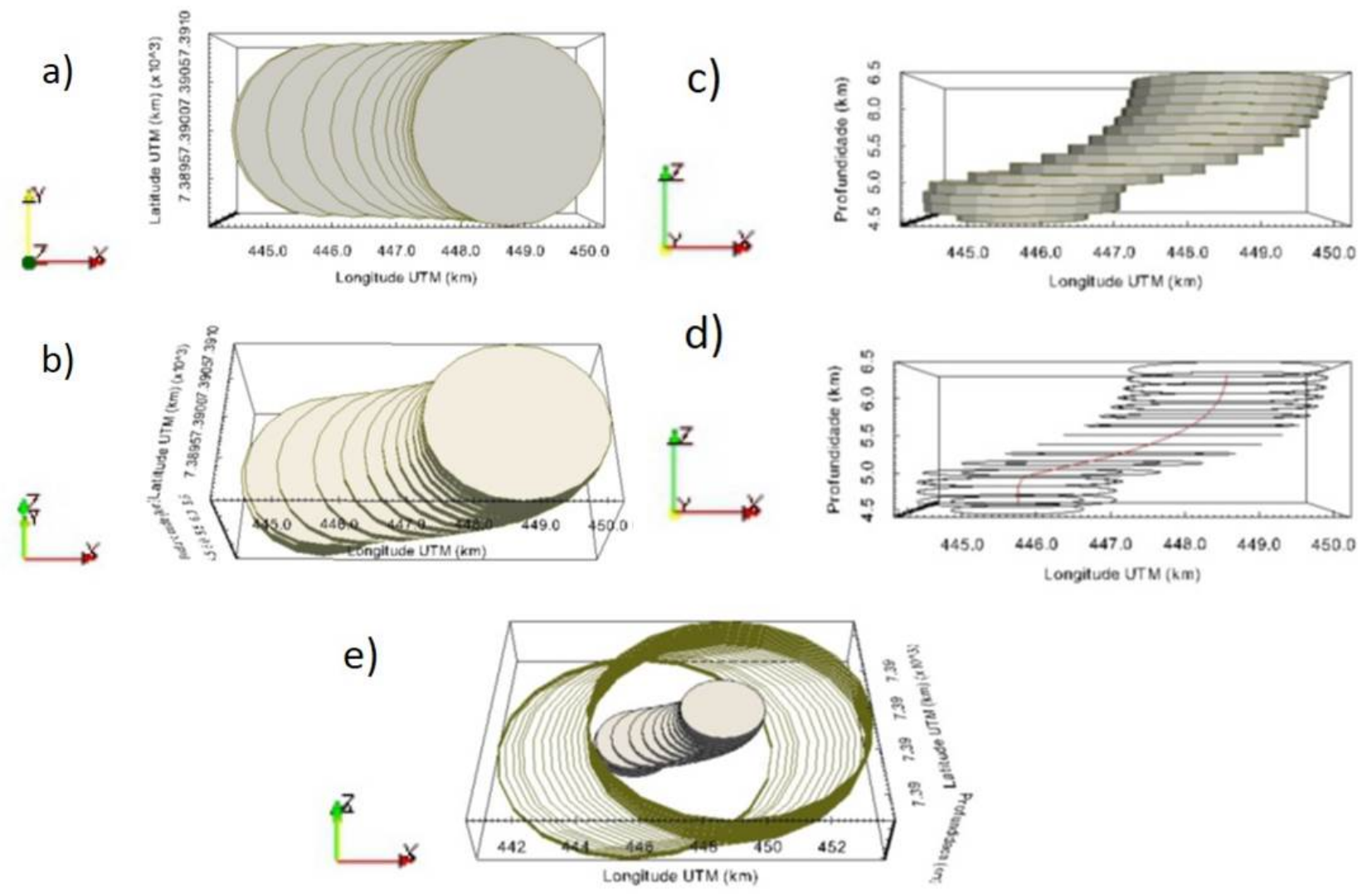

Figura 11: Visualização da geometria do paredão de sal 1 estimada pelo modelo de inversão gravimétrica 3D a partir de 15 prismas com 20 vértices cada, $\Delta \rho$ de -0.46 g. $\mathrm{cm}^{-3}$, distância radial inicial do corpo de $1.5 \mathrm{~km}$, variações possíveis das distâncias radiais entre 1.0 e $2.0 \mathrm{~km}$ e profundidades desde $4.4 \mathrm{~km}$ até $6.5 \mathrm{~km}$. O corpo modelado é apresentado em (a) paralelo aos eixos $x$ e $y$ e perpendicular ao eixo $z$, em (b) o corpo está rotacionado em aproximadamente $60^{\circ}$ em relação ao eixo $x$, em (c) paralelo ao eixo $z$ e perpendicular aos eixos $x$ e $y$, em (d) observa-se a evolução das coordenadas iniciais dos prismas que compõem o corpo de sal (linha vermelha) e em (e) é feita uma comparação do que seria o verdadeiro tamanho do paredão de sal (esqueleto externo) com o previsto pelo modelo (corpo maciço interno).

A figura 11 mostra os resultados do que seria a geometria do corpo de sal do caso 1 , enquanto que a figura 12 mostra a validação dos testes realizados a partir das sobreposições das isogais observadas e preditas pelo modelo. A partir da figura 3, estimou-se como informação a priori uma distância radial inicial de $4.0 \mathrm{~km}$, com variações das distâncias radiais entre 4.0 e 6.0 $\mathrm{km}$. Entretanto, o melhor ajuste entre as isogais preditas e observadas se dá quando subestimamos as distâncias radiais possíveis para a modelagem do corpo de sal (distância radial inicial de $1.5 \mathrm{~km}$ e variações das distâncias radiais entre 1.0 e $2.0 \mathrm{~km}$ - figura 12a). Para ambos os casos (tamanhos do paredão de sal real e subestimado), o modelo gerou geometrias semelhantes (figura 11e). A figura 11 mostra o paredão de sal alongado para o leste, com maior espessura na parte leste e menor espessura na parte oeste; e profundidades variando desde $4.4 \mathrm{~km}$ até 6.5 km (informação a priori obtida na figura 3). Comparando as figuras 3 e 11, nota-se um déficit de massa gerado pelo modelo na base do corpo de sal, na parte leste do mesmo. 

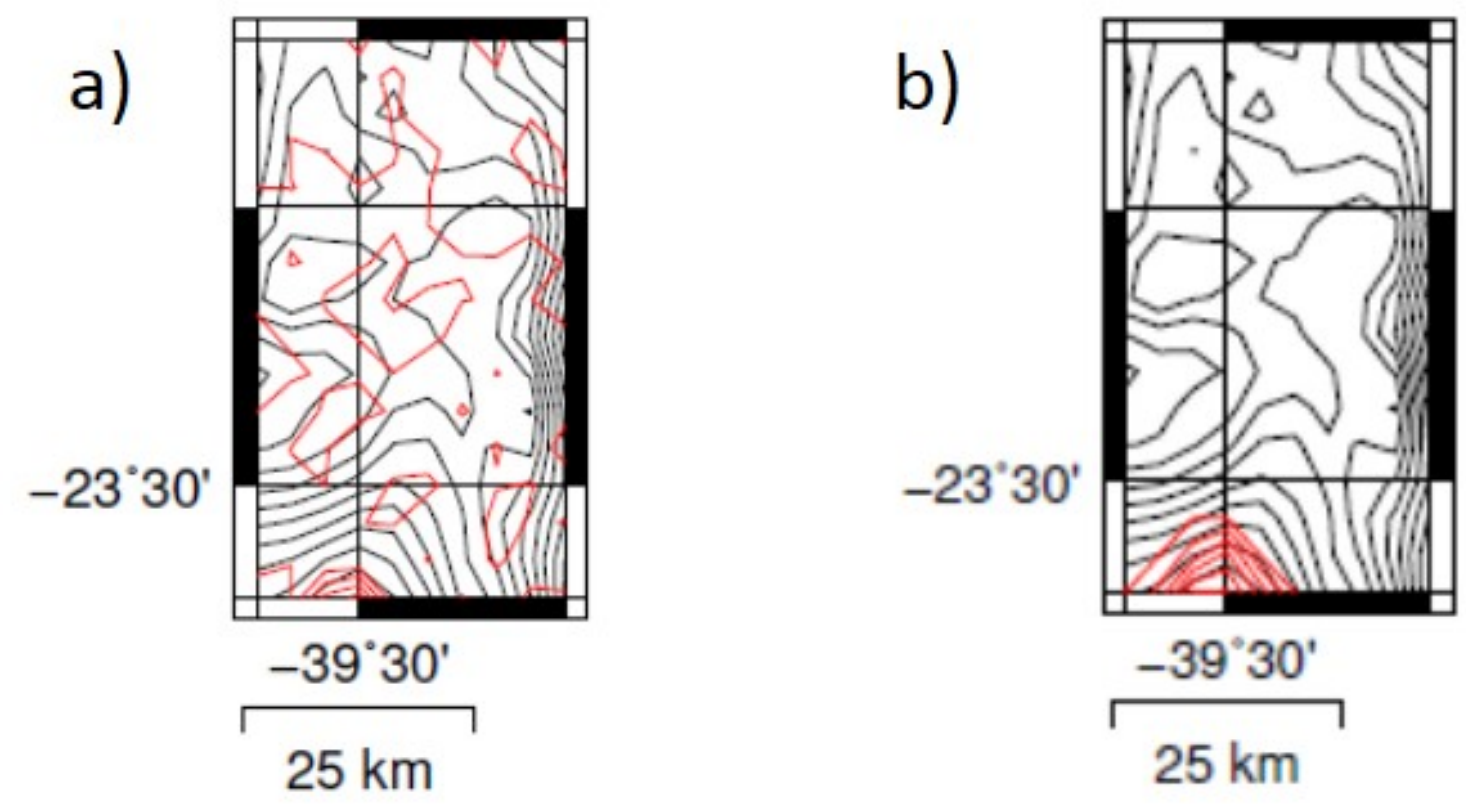

Figura 12: Sobreposição das isogais das anomalias Bouguer residuais observadas (linhas pretas) e preditas pelo modelo (linhas vermelhas) utilizando como parâmetros de entrada distâncias radiais iniciais de (a) 1.5 km (variações entre variações das distâncias radiais entre 1.0 e $2.0 \mathrm{~km}$ ) e (b) $4.0 \mathrm{~km}$ (variações entre variações das distâncias radiais entre 4.0 e $6.0 \mathrm{~km}$ ). $\mathrm{O}$ intervalo de contornos das anomalias observadas (preditas) é de 1(10) mGal.

\section{Modelagem do corpo 2}

Nesta parte do trabalho, serão descritos os resultados do modelo de inversão gravimétrica 3D para o paredão de sal que apresenta anomalia residual Bouguer fechada (caso 2), classificado como paredão 2. A coordenada inicial a partir da qual o corpo de sal começará a ser construído foi definida por um ponto de mínimo; no caso, o segundo mínimo absoluto da anomalia residual Bouguer (quadrado vermelho da figura 13). As coordenadas UTM do ponto de mínimo são (493.184, 7419.544). As coordenadas dos vínculos de desigualdade ou os limites para a modelagem (triângulos verdes da figura 13) são: (489.779, 7417.851) sendo estas as coordenadas do terceiro mínimo absoluto; e a outra (500, 7426.926).

A figura 14 mostra o paredão de sal 2 alongado para nordeste e profundidades variando entre $4.4 \mathrm{~km}$ e $6.0 \mathrm{~km}$. Tal geometria obtida pelo modelo para o paredão de sal 2 é razoável, visto que a figura 15 mostra que as sobreposições das isogais observadas e preditas pelo modelo foram satisfatórias. A distância radial do primeiro prisma foi de $3.0 \mathrm{~km}$, com variações possíveis das distâncias radiais entre $2.78 \mathrm{~km}$ e $12 \mathrm{~km}$. Assim como no caso do paredão de sal 1 , o modelo mostra o que parece ser um déficit de massa na parte leste da base do paredão, quando comparamos as figuras 3 e 14 .

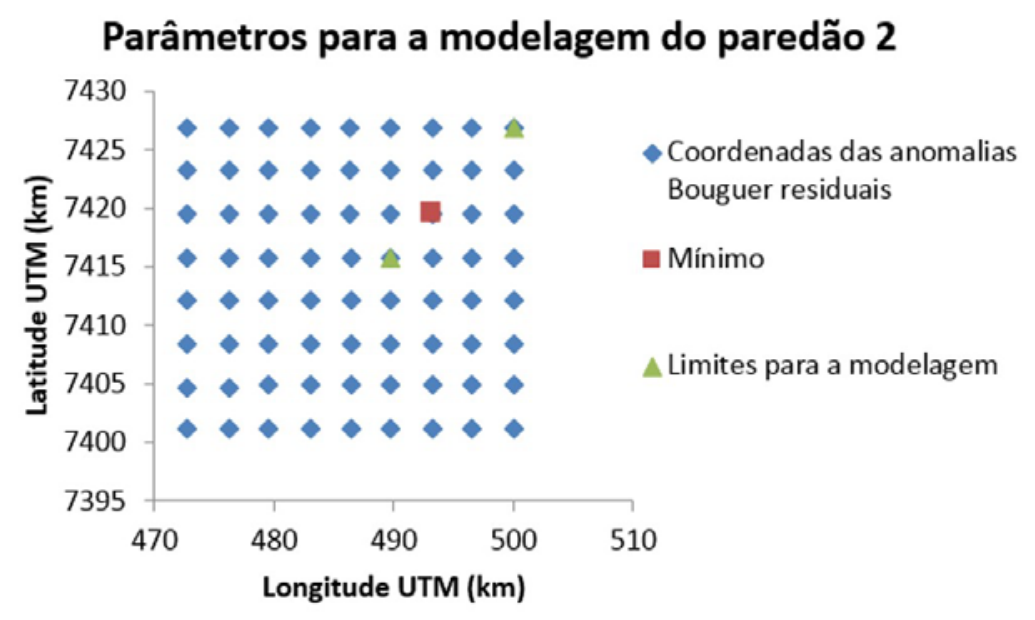

Figura 13: Ponto de mínimo $\left(x_{0}, y_{0}\right)$ a partir do qual os prismas (paredão de sal 2) começarão a ser construídos (quadrado vermelho) e coordenadas limites para a modelagem ou vínculos de desigualdade (triângulos verdes). 
a)

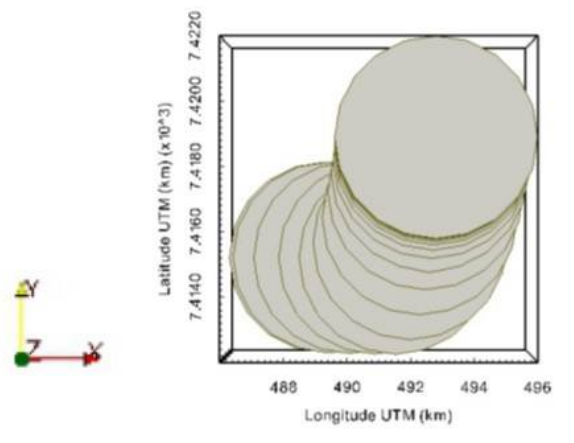

c)

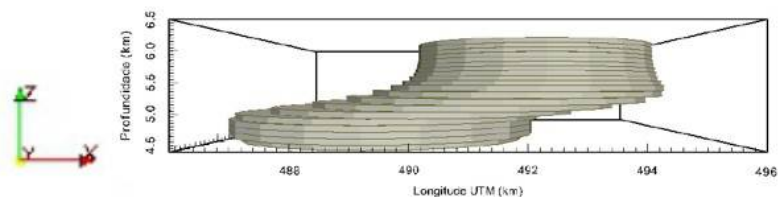

b)

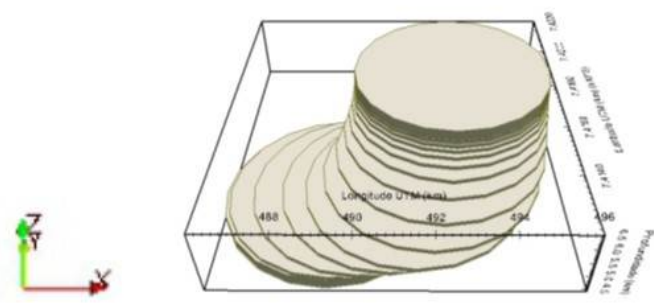

d)

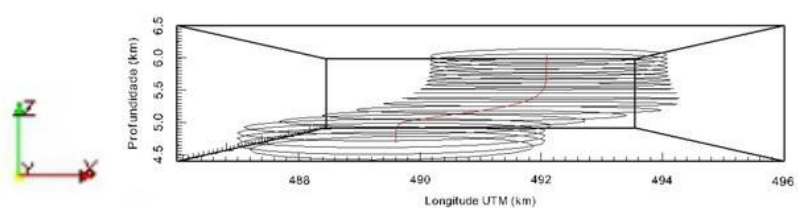

Figura 14: Visualização da geometria do paredão de sal 2 estimada pelo modelo de inversão gravimétrica 3D a partir de 20 prismas com 20 vértices cada, $\Delta \rho$ de $-0.46 \mathrm{~g} . \mathrm{cm}^{-3}$, distância radial inicial do corpo de $3 \mathrm{~km}$, variações possíveis das distâncias radiais entre 2.78 e $12 \mathrm{~km}$ e profundidades desde $4.4 \mathrm{~km}$ até $6.5 \mathrm{~km}$. O corpo modelado é apresentado em (a) paralelo aos eixos $x$ e $y$ e perpendicular ao eixo $z$, em (b) o corpo está rotacionado em aproximadamente $60^{\circ}$ em relação ao eixo $x$, em (c) paralelo ao eixo $z$ e perpendicular aos eixos $x$ e $y$ e em (d) observa-se a evolução das coordenadas iniciais dos prismas que compõem o corpo de sal (linha vermelha).

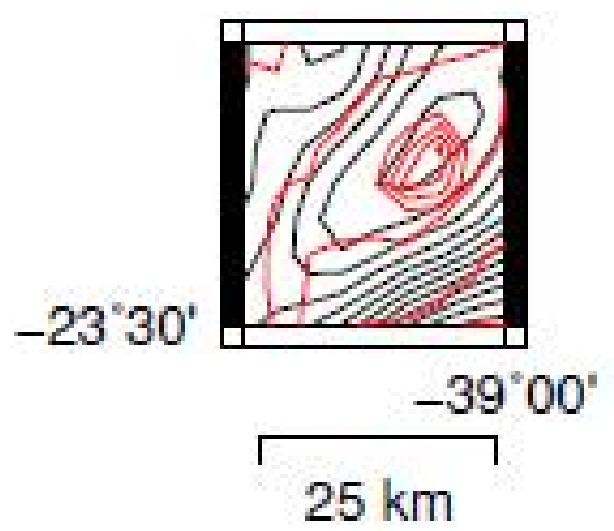

Figura 15: Sobreposição das isogais das anomalias Bouguer residuais observadas (linhas pretas) e preditas pelo modelo (linhas vermelhas). O intervalo de contornos das anomalias observadas (preditas) é de 2 (8) mGal.

\section{CONCLUSÕES}

Foram modelados paredões de sal Um problema observado quando comparadas considerando dois casos distintos: uma anomalia residual Bouguer aberta (caso 1) e outra fechada (caso 2). As geometrias dos paredões de sal foram modeladas satisfatoriamente em ambos os casos. Aparentemente, o modelo gerou um déficit de massa na parte leste de ambos os paredões de sal, como se observa na figura 16. Entretanto, não se pode dizer que seja um erro gerado pelo modelo, pois a própria seção sísmica da região se mostra duvidosa quanto à configuração real das bases dos paredões de sal. $\mathrm{O}$ ajuste entre as isogais observadas e preditas também se mostrou satisfatório em ambos os casos (melhor no caso 2). as modelagens dos paredões de sal no caso 1 e no caso 2 foi que o tamanho do paredão no caso 1 teve que ser subestimado para se obter um melhor ajuste entre as isogais observadas e preditas. No caso 2 não foi observado este problema. Conclui-se que o modelo de inversão gravimétrica 3D parece ser mais eficaz em modelar anomalias Bouguer fechadas do que abertas. Os resultados do modelo mostram que estudos de gravimetria em bacias sedimentares com potencial petrolífero podem ser úteis para estabelecer os volumes relativos das rochas de sal utilizando contrastes de densidade conhecidos. 
(a)

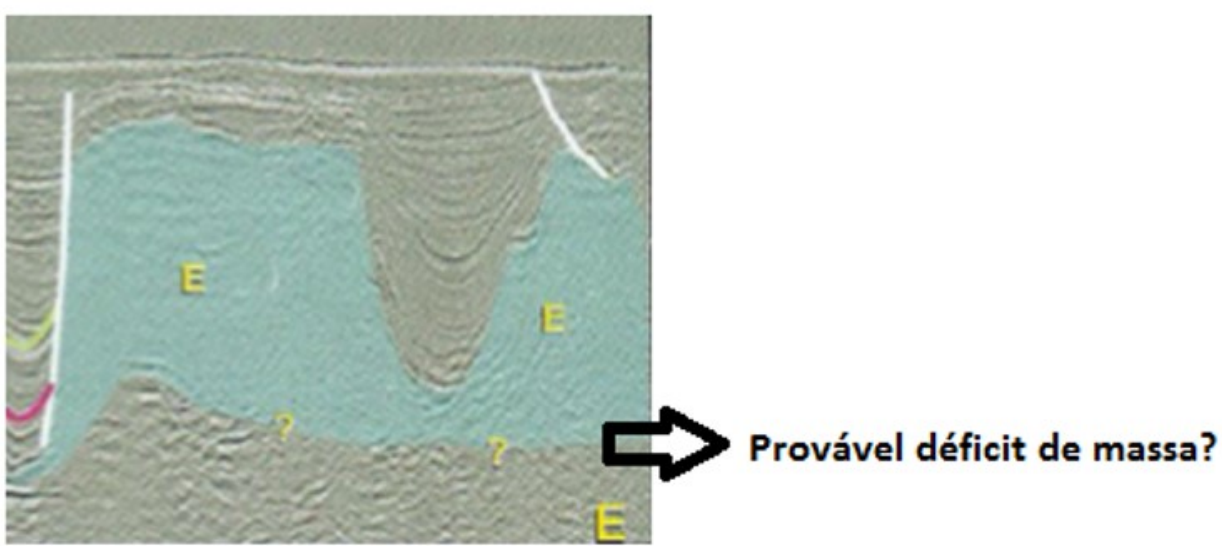

(b)

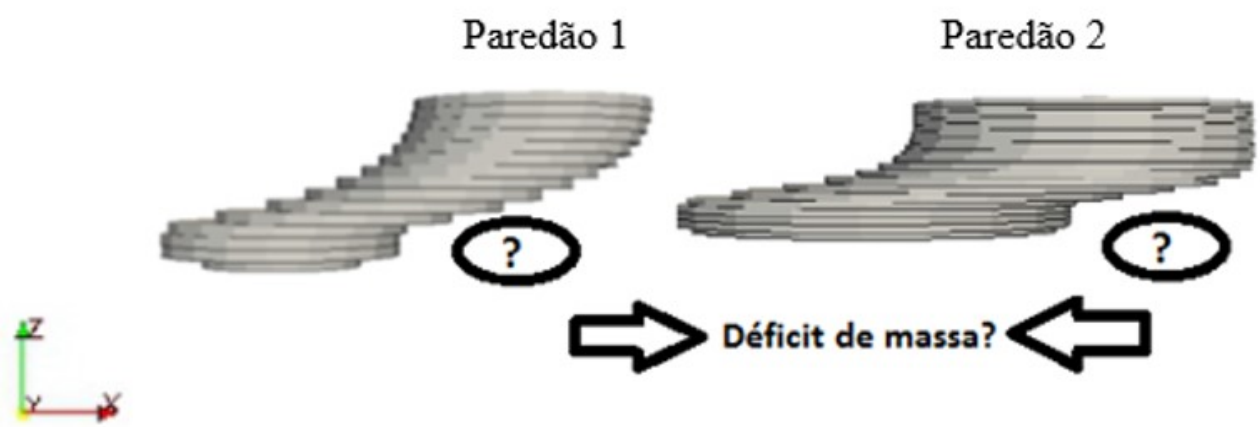

Figura 16: (a) Comparação entre as geometrias dos paredões de sal obtidos por sísmica de reflexão - MOHRIAK et al. (2008) e (b) modeladas pelo modelo 3D de inversão gravimétrica.

\section{REFERÊNCIAS}

BARBOSA, V. C. F.; SILVA, J. B. C. Generalized compact gravity inversion. Geophysics, v. 59, n. 1, p. 57-68, 1994.

BARBOSA, V. C. F.; SILVA, J. B. C; MEDEIROS, W. E. Gravity inversion of basement relief using approximate equality constraints on depths. Geophysics, v. 62, n. 6, p. 1745-1757, 1997.

BEAR, G. W.; AL-SHUKRI, H. J.; RUDMAN, A. J. Linear inversion of gravity data for 3-D density distributions. Geophysics, vol. 60, n. ${ }^{\circ}$ 5, p. 1354-1364, 1995.

BLAKELY, R. J. Potential theory in gravity and magnetic applications. Cambridge University Press, 1996.

CONTRERAS, J.; ZUHLKE, R.; BOWMAN, S.; BECHSTADT, T. Seismic stratigraphy and subsidence analysis of the southern Brazilian margin (Campos, Santos and Pelotas basins). Marine and Petroleum Geology, v. 27, n. 9, p. 1952-1980, 2010.

CORDELL, L.; HENDERSON, R. G. Iterative threedimensional solution of gravity anomaly data using a digital computer. Geophysics, v. 33, n. 4, p. 596-601, 1968.

DE MELO GARCIA, S. F., LETOUZEY, J., RUDKIEWICZ, J. L., DANDERFER FILHO, A., DE LAMOTTE, D. F.. Structural modeling based on sequential restoration of gravitational salt deformation in the Santos Basin (Brazil). Marine and Petroleum Geology, v. 35, n. 1, p. 337-353, 2012.

DEMERCIAN, S.; SZATMARI, P.; COBBOLD, P. R. Style and pattern of salt diapirs due to thin-skinned gravitational gliding, Campos and Santos basins, offshore Brazil. Tectonophysics, v. 228, n. 3-4, p. 393-433, 1993.

DUTRA, A. C.; MARANGONI, Y. R. Gravity and magnetic 3D inversion of Morro do Engenho complex, Central Brazil. Journal of South American Earth Sciences, v. 28, n. 2, p. 193-203, 2009.
KRAHENBUHL, R. A.; LI, Y. Inversion of gravity data using a binary formulation. Geophys. J. Int., 167, p. 543-556, 2006.

LI, Y.; OLDENBURG, D. W. 3-D inversion of gravity data. Geophysics. vol. 63, n. ${ }^{\circ}$ 1, p. 109-119, 1998.

MOHLIAK, W. U.; DEWEY, J. F. Deep seismic reflectors in the Campos basin, offshore Brazil. Geophysical Journal International, v. 89, n. 1, p. 133-140, 1987.

MOHRIAK, W. U.; HOBBS, R.; DEWEY, J. F: Basin-forming processes and the deep structure of the Campos Basin, offshore Brazil. Marine and Petroleum Geology, v. 7, n. 2, p. 94IN1101-100IN4122, 1990.

MOHRIAK, W., SZATMARI, P., ANJOS, S. M. C. SAL: Geologia e Tectônica - Exemplos nas Bacias Brasileiras. Segunda edição. São Paulo: Beca Edições Ltda, 2008.

NAGIHARA, S.; HALL, S. A. Three-dimensional gravity inversion using simulated annealing: Constraints on the diapiric roots of allochthonous salt structures. Geophysics, vol. 66, n. ${ }^{\circ}$ 5, p. 1438-1449, 2001.

ODÉ, H. Review of mechanical properties of salt relating to salt dome genesis. Geological Society of America Special Papers, v. 88, p. 543-596, 1968.

OEZSEN, R. Velocity modeling and prestack depth imaging below complex salt structures: a case history from on-shore Germany. Geophysical Prospecting, vol. 52, p. 693-705, 2004.

OJEDA, H. A. O. Structural framework, stratigraphy, and evolution of Brazilian marginal basins. AAPG Bulletin, v. 66, n. 6, p. 732-749, 1982.

OLIVEIRA JR., V.C. Inversão gravimétrica radial por camadas para reconstrução de corpos geológicos 3D. Observatório Nacional, Rio de Janeiro, Dissertação de Mestrado, 135 pp, 2010. 
OLIVEIRA JR.,V.C e L. UIEDA. Tópicos de Inversão Geofísica- XIV Escola de Verão de Geofísica 2012 - IAG, USP, 2012.

OLIVEIRA, V. C.; BARBOSA, V. C. F. 3-D radial gravity gradient inversion. Geophysical Journal International, v. 195, n. 2, p. 883-902, 2013.

RUGGIERO, M. A. G., LOPES, V. L.R. Cálculo numérico: aspectos teóricos e computacionais. Makron Books do Brasil, 1997.

SANDWELL, D. T., R. D. MÜLLER, W. H. F. SMITH, E. GARCIA, R. FRANCIS. New global marine gravity model from CryoSat-2 and Jason-1 reveals buried tectonic structure, Science, Vol. 346, no. 6205, pp. 65-67, doi: 10.1126/science.1258213, 2014.

SANDWELL, D. T., W. H. F. SMITH. Global marine gravity from retracked Geosat and ERS-1 altimetry: Ridge Segmentation versus spreading rate, J. Geophys. Res., 114, B01411, doi:10.1029/2008JB006008, 2009.

SANDWELL, D. T., E. GARCIA, K. SOOFI, P. WESSEL, AND W. H. F. SMITH. Towards $1 \mathrm{mGal}$ Global Marine Gravity from CryoSat-2, Envisat, and Jason-1, The Leading Edge, 32(8), 892-899. doi: 10.1190/tle32080892.1, 2013

SILVA, J. B. C.; BARBOSA, V. C. F. Generalized radial inversion of 2D potential field data. Geophysics. vol. 69, n. ${ }^{\circ}$ 6, p. 1405-1413, 2004.
SILVA, J. B. C.; BARBOSA, V. C. F. Interactive gravity inversion. Geophysics, v. 71, n. 1, p. J1-J9, 2006.

SILVA DIAS, F.J.S.; BARBOSA, V.C.F.; SILVA, J.B.C.. Adaptive learning 3D gravity inversion for salt-body imaging. Geophysics vol. 76, n. ${ }^{\circ}$. 3, p. I49/I57, 2011.

SMITH, W. H. F., D. T. SANDWELL. Global seafloor topography from satellite altimetry and ship depth soundings, Science, v. 277, p. 1957-1962, 26 Sept., 1997.

STARICH, P. J.; LEWIS, G. G.; FAULKENR, J.; STANDLEY, P. G. ; SETTERQUIST, S. Integrated geophysical study of an onshore salt dome. The Leading Edge, vol. 13, p. 880-884, 1994.

TALWANI, M., WORZEL, J. L., LANDISMAN, M.. Rapid gravity computations for two dimensional bodies with application to the Mendoncino submarine fracture zonel. J. Geophys. Res. vol. 64, 49-59, 1959.

TELFORD, W. M.; GELDART, L. P.; SHERIFF, R. E. Applied geophysics. Cambridge university press, 1990.

WESSEL, P., W. H. F. SMITH. The Generic Mapping Tools, version 4.3.1, technical reference and cookbook, 212 p, 2008.

Submetido em 10 julho de 2017 Aceito em 28 de dezembro de 2018 